\title{
Disentangling component spectra of $\kappa$ Sco, a spectroscopic binary with a pulsating primary ${ }^{\star}$
}

\section{Interpretation of the line-profile variability}

\author{
K. Uytterhoeven ${ }^{1}$, M. Briquet ${ }^{1, \star \star}$, C. Aerts ${ }^{1,2}$, J. H. Telting ${ }^{3}$, P. Harmanec ${ }^{4,5}$, K. Lefever ${ }^{1}$, and J. Cuypers ${ }^{6}$ \\ 1 Institute of Astronomy, Katholieke Universiteit Leuven, Celestijnenlaan 200 B, 3001 Leuven, Belgium \\ e-mail: Katrien.Uytterhoeven@ster.kuleuven.ac.be \\ 2 Department of Astrophysics, University of Nijmegen, PO Box 9010, 6500 GL Nijmegen, The Netherlands \\ 3 Nordic Optical Telescope, Apartado de Correos 474, 38700 Santa Cruz de La Palma, Spain \\ 4 Astronomical Institute of the Charles University, V Holešovičkách 2, 18000 Praha 8, Czech Republic \\ 5 Astronomical Institute, Academy of Sciences, 25165 Ondřejov, Czech Republic \\ ${ }^{6}$ Royal Observatory of Belgium, Ringlaan 3, 1180 Brussel, Belgium
}

Received 10 June 2004 / Accepted 31 October 2004

\begin{abstract}
We analyse the complex short-term Si III line-profile variability of the spectroscopic binary $\beta$ Cep star $\kappa$ Sco after orbit subtraction, before and after spectral disentangling. We refine the known oscillation frequency of the star: $f_{1}=4.99922 \mathrm{c} \mathrm{d}^{-1}$ and detect $2 f_{1}$. Variability is also found at frequencies near $f_{2} \simeq 4.85 \mathrm{c} \mathrm{d}^{-1}$ and $f_{3} \simeq 5.69 \mathrm{c} \mathrm{d}^{-1}$ or their aliases. These frequencies are not significant if we consider the spectra alone, but they survive our selection after the consideration that they were derived previously from independent ground-based and space photometry by different teams. Moreover, we find dominant variability in the equivalent width with a frequency in the interval $[0.22,0.30] \mathrm{c} \mathrm{d}^{-1}$ which we interpret as the rotational frequency $f_{\text {rot }}$ of the star. The complex window function does not allow us to determine definite values for $f_{2}, f_{3}, f_{\text {rot }}$. The variability with $f_{1}$ is interpreted as a prograde non-radial oscillation mode with spherical wavenumbers $(\ell, m)=(2,-1)$ or $(1,-1)$. The additional frequencies are explained in terms of rotational modulation superposed to the main oscillation. We also point out that we cannot disprove the variability in $\kappa$ Sco to originate from co-rotating structures. KOREL disentangling preserves the large-amplitude line-profile variability but its performance for complex low-amplitude variability remains to be studied in detail.
\end{abstract}

Key words. binaries: spectroscopic - stars: oscillations - line: profiles - stars: individual: $\kappa$ Sco

\section{Introduction}

Significant progress in the modelling of the internal stellar structure of $\sim 10 M_{\odot}$ stars was achieved recently by Aerts et al. (2003a) for the star HD 129929 and by Pamyatnykh et al. (2004) for the star HD 29248. Through seismic modelling of their multiplet non-radial pulsation (NRP) frequencies, it was shown that the overshooting parameter is less than 0.15 times the pressure scale height and that non-rigid rotation is present, with the rotation near the core about 3 times faster than the surface rotation. It is remarkable that these two stars have such a similar internal structure and a natural question is if more rapid rotators have the same internal behaviour.

* Based on observations obtained with the Coudé Échelle Spectrograph on the ESO CAT telescope and with the CORALIE échelle spectrograph on the 1.2-m Euler Swiss telescope, both situated at La Silla, Chile.

$\star \star$ Postdoctoral fellow of the Fund for Scientific Research of Flanders (FWO).
From their list of 26 confirmed line-profile (LP) variable $\beta$ Cep stars, Aerts \& De Cat (2003) found a quite clear separation between slow $\left(v \sin i<40 \mathrm{~km} \mathrm{~s}^{-1}\right)$ and moderate $(v \sin i>$ $80 \mathrm{~km} \mathrm{~s}^{-1}$ ) rotators. Most of the moderate rotators are members of a spectroscopic binary. In this paper, we report on the intrinsic variations in one of these moderate rotators among the $\beta$ Cep stars with the hope to provide sufficient frequency information for future seismic modelling.

In a recent study of the bright B $1.5 \mathrm{III}$ star $\kappa$ Sco (HD 160578, HR 6580, HIP 86670, $V=2^{\mathrm{m}} .4$ ), Harmanec et al. (2004, hereafter termed Paper I) applied KOREL ${ }^{1}$ (Hadrava 1995) disentangling to derive the orbital parameters and to unravel the contributions of both components to the line-profile variability (LPV) observed in the Si III 4552 and $4567 \AA$ lines of a set of 699 spectra. The binary has an

\footnotetext{
1 The program is freely available to interested users. Its detailed description, Fortran source code and user's guide can be obtained via http://www.asu.cas.cz/ had/had.html upon registering at e-mail: had@sunstel.asu.cas.cz
} 


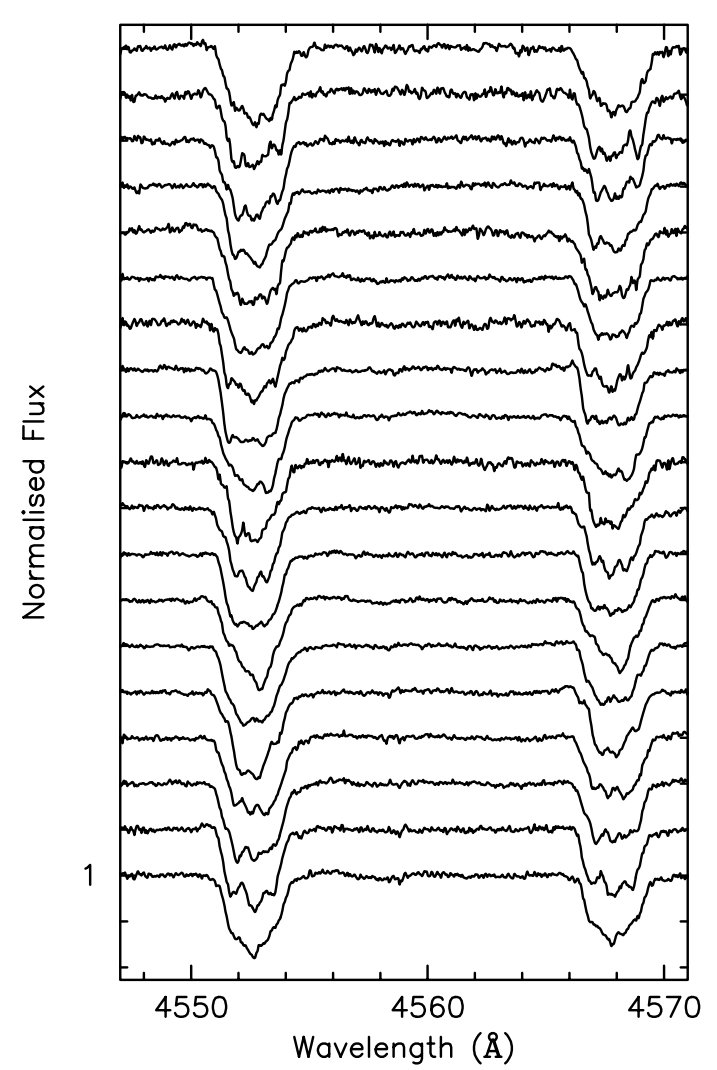

Fig. 1. A randomly chosen set of the normalised disentangled Si III profiles of $\kappa$ Sco (HJDs 2449876-50 658) in the rest frame of the primary centered at $4560 \AA$. For clarity we have shifted the flux level of each profile with a value 0.04 with respect to the previous one.

eccentric orbit ( $e=0.50$ ) with a period of some 196 days. We refer to Paper I for details and for a complete history of the variability studies of this star, as well as for the first evaluation of the KOREL disentangling procedure. One of the important findings of Paper I was that only the primary is a LP variable.

In the current paper we investigate in detail the LPV of the primary of $\kappa$ Scorpii, based on 699 profiles obtained with and without KOREL disentangling. Some observed profiles are shown in Fig. 1. In Paper I we showed that the dominant intrinsic frequency could be recovered from the disentangled profiles in the rest frame of the primary. We recall that the synodic frequency $4.99922 \mathrm{c} \mathrm{d}^{-1}$ (called $f_{1}$ hereafter) was found in the residuals of the radial velocities after orbit subtraction, while the sidereal frequency $5.00425 \mathrm{c} \mathrm{d}^{-1}$, known from groundbased photometry (Lomb \& Shobbrook 1975) and recovered in the WIRE photometry (Cuypers et al. 2004), was detected in the KOREL line-strength variations, whereby the difference between the two frequencies is exactly equal to the orbital frequency. In this paper we elaborate further on the profiles with the goal to interpret their variations in terms of NRP modes, given that the star is a confirmed $\beta$ Cep variable. Shobbrook \& Lomb (1972) and Lomb \& Shobbrook (1975) classified the star as such after their discovery of the frequency $5.00425 \mathrm{c} \mathrm{d}^{-1}$ in the light changes. These authors also suspected the presence of the frequency $4.86784 \mathrm{c} \mathrm{d}^{-1}$ (hereafter called $f_{2}$ ) as a result of a beat-phenomenon. Uytterhoeven et al. (2001) already showed the presence of these two frequencies in the LPV of the subset of 422 spectra gathered during 8 consecutive nights of continuous monitoring of the star, without having disentangled the lines of the primary in these spectra. We improve their results here by considering all the 699 available LP data. In particular we aim to search in our spectroscopic data for the presence of another frequency of $5.2767 \mathrm{c} \mathrm{d}^{-1}$ (hereafter called $f_{4}$ ) suggested by Lomb \& Shobbrook (1975) and three additional frequencies recently found by Cuypers et al. (2004) in white-light photometry gathered by the WIRE satellite: $5.6950 \mathrm{c} \mathrm{d}^{-1}\left(f_{3}\right)$, $2.2498 \mathrm{c} \mathrm{d}^{-1}\left(f_{5}\right)$ and $0.2809 \mathrm{c} \mathrm{d}^{-1}$.

\section{Physical parameters of the system}

The basic physical parameters of both components were derived in Paper I from the orbital solution and from photometric and parallax results available in the literature. In order to have an independent check of them, and to derive the flux contribution of the secondary, we observed $\kappa$ Sco twice with the CORALIE échelle spectrograph attached to the $1.2 \mathrm{~m}$ Leonard Euler telescope in La Silla, Chile during one night in March 2004. Both spectra were reduced following the standard procedure and were co-added to obtain the highest possible signal-to-noise ratio $(S / N=195)$.

To derive good estimates of $T_{\text {eff }}$ and $\log g$ of both stars we focused on the regions of the Si II (4128 $\AA, 5050 \AA)$, Si III (4560 ̊, $4820 \AA, 4716 \AA, 5739 \AA)$, Si IV (4116, $4089 \AA), \mathrm{He}$ I (4026 ̊, $4387 \AA, 4471 \AA, 4922 \AA, 4713 \AA$, $6678 \AA$ ), He II (4686 $⿱$ ) $, \mathrm{H} \alpha, \mathrm{H} \beta, \mathrm{H} \gamma, \mathrm{H} \delta$ and $\mathrm{H} \epsilon$ line profiles. These observed lines were compared with theoretical ones computed for spherically symmetric non-LTE atmosphere models by means of the newest version of the FASTWIND code (Santolaya-Rey et al. 1997). The input parameters to obtain the theoretical profiles are $T_{\text {eff }}, \log g$, the microturbulent velocity and the particle number ratio $n(\mathrm{He}) / n(\mathrm{H})$. The gravity and temperature are most constrained by respectively the wings of the Balmer lines and the comparison between the Si II, Si III, Si IV lines. We restricted to atmospheres with solar helium composition.

There is only one He II line present in the spectrum and it is extremely weak. It concerns the He II $4686 \AA$ line, which is indeed the first such line that occurs as the temperature raises above $24000 \mathrm{~K}$. The other He II lines (e.g. the ones at $6406 \AA$, $6527 \AA$ and $6683 \AA$ ) are absent. This implies an upper limit of $25000 \mathrm{~K}$ for the effective temperature of the primary, which was the estimate derived in Paper I. Further, no Si IV lines are detected, while the Si III lines are strong. The Si II doublet at $4128 \AA$ and $4130 \AA$, while severely blended due to the rotation, is clearly present. Fitting the lines for a single star with $T_{\text {eff }}=25000 \mathrm{~K}$ gives too strong Si III profiles and too weak Si II lines. We therefore conclude that the secondary has a significant contribution to the Si III lines and is mainly responsible for the Si II lines. The Balmer wings, on the other hand, lead us to $\log g$ between 3.6 and 4.0 assuming a single star.

We subsequently merged the lines of two contributing stars according to different flux ratios using the relative orbital velocity of the components and the $v \sin i$ estimates derived in Paper I. The best overall fit to observed line profiles occurs for 
HJD 2450742.4691
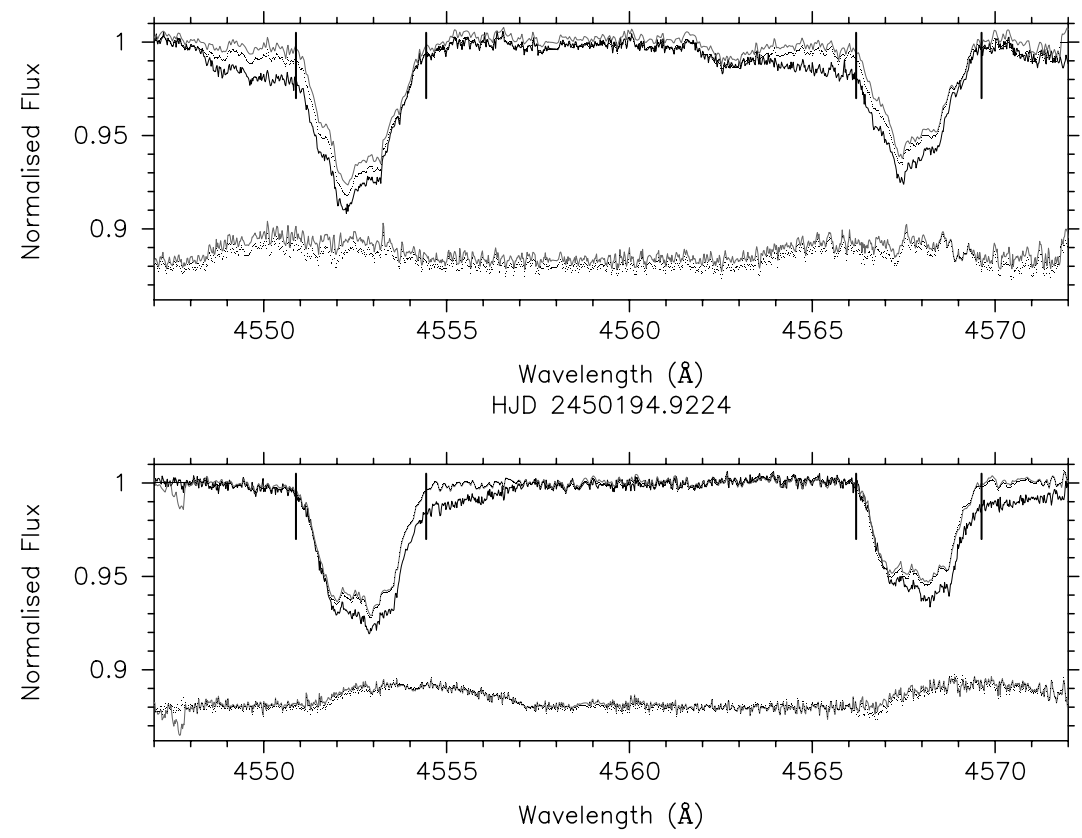

Fig. 2. Top (bottom): line profile measurements taken on HJD 2450742.4691 (HJD 2450 194.9224). The observed spectrum of the primary shifted with $+53.39 \mathrm{~km} \mathrm{~s}^{-1}\left(-55.34 \mathrm{~km} \mathrm{~s}^{-1}\right)$ is given by the full black line and the KOREL disentangled profile in the rest frame of the primary by the dotted line. The gray profile is the observed one from which the disentangled profile of the secondary is subtracted after applying its orbital shift of $-57.84 \mathrm{~km} \mathrm{~s}^{-1}\left(62.70 \mathrm{~km} \mathrm{~s}^{-1}\right)$ and to which a subsequent orbital shift towards the rest frame of the primary $+53.39 \mathrm{~km} \mathrm{~s}^{-1}\left(-55.34 \mathrm{~km} \mathrm{~s}^{-1}\right)$ is applied. The vertical lines indicate the integration boundaries we used to calculate the velocity moments from the Si III profiles centered at $4552.654 \AA$ and $4567.872 \AA$. At the bottom of each figure the residual disentangled and secondary removed spectra with respect to the observed spectrum are also shown, after an arbitrary shift towards flux level 0.88 .

the following parameters: $T_{\mathrm{eff}}=25000 \mathrm{~K}, \log g=3.8$ for the primary and $T_{\mathrm{eff}}=20000 \mathrm{~K}, \log g=3.8$ for the secondary. The uncertainties are $2000 \mathrm{~K}$ for the temperature and 0.2 dex for the gravity, for both stars. These results are entirely compatible with those of Paper I. They lead to a ratio of the flux of the primary to the one of the secondary between 1.5 and 2.5.

\section{Disentangled versus non-disentangled spectra}

We refer to Paper I for a detailed description of the 699 profiles used in this study. The data have a time span of some 3 years, including two intensive monitoring campaigns lasting 3 and 8 nights. By means of the KOREL disentangling procedure the spectrum of the spectroscopic binary was decomposed in the following parts: the disentangled profile of the primary, the disentangled profile of the secondary and the residual spectrum calculated in either the rest frame of the primary or the rest frame of the secondary. As already pointed out in Paper I, the KOREL code is based on the assumption that the line profiles do not change shape, a condition that is evidently violated in the current case of the primary of $\kappa$ Sco. One of the main goals of this paper is to investigate what the implications of this violation are on the interpretation of the LPV.

Before Paper I, KOREL disentangling was not yet applied as an intermediate step in the interpretation of LPV due to oscillations. Therefore, we carefully checked its performance by employing three different procedures to analyse the intrinsic variability of the primary. In the first procedure we consider the "original profiles" and simply shift them to the rest frame of the primary, i.e. we ignore the contribution of the secondary to the line. KOREL provides the disentangled spectra with an unknown shift of the continuum (Hadrava 1997). For the second procedure, we normalised (i.e. re-rectified) the disentangled spectrum of the primary produced by KOREL before coadding the residuals. Hereafter we will refer to these profiles as the "disentangled profiles". In the third procedure we shift the normalised KOREL disentangled profile of the secondary according to its orbital velocity, we subtract it from the observed profile assuming equal fluxes of both components and we finally move this resulting profile to the rest frame of the primary. These profiles will be referred to as the "secondary subtracted profiles". We thus incorporate the two extreme cases that the secondary has no and equal contributions to the observed flux while KOREL does not take into account any flux ratio information.

In Fig. 2 we make a comparison between the profiles resulting from these three different types of numerical operation for opposite elongations. We also give the residual disentangled and secondary removed spectra with respect to the original spectrum. One can see that the KOREL disentangled profile of the primary and the one obtained after subtraction of the secondary's profile differ only slightly in line depth and not in shape of the line features. This implies that KOREL's solution is not too different from the one obtained assuming equal fluxes for the Si III triplet, while we found a flux ratio between 1.5 and 2.5 from the échelle spectrum in the previous section. 
Table 1. Amplitudes (in $\mathrm{km} \mathrm{s}^{-1}$ ) of the frequencies $f_{\text {rot }} \in[0.22 ; 0.28] \mathrm{c} \mathrm{d}^{-1}, f_{1}=4.99922 \mathrm{c} \mathrm{d}^{-1}$ and $2 f_{1}$ for the $E W$ (top); amplitudes (in km s${ }^{-1}$ ) of $f_{1}, f_{2}, f_{3}$ and $2 f_{1}$ for $\langle v\rangle$ (bottom) of the Si III $4552.654 \AA$ (left) and Si III $4567.872 \AA$ (right) line of $\kappa$ Sco, calculated from the original observed spectra and from the disentangled spectra. The last column denotes the total variance reduction (f.v.) derived from the least-squares fits to the data with the considered frequencies.

\begin{tabular}{|c|c|c|c|c|c|c|c|c|}
\hline & \multicolumn{4}{|c|}{$4552.654 \AA$} & \multicolumn{4}{|c|}{$4567.872 \AA$} \\
\hline & \multicolumn{2}{|c|}{ Original } & \multicolumn{2}{|c|}{ Disentangled } & \multicolumn{2}{|c|}{ Original } & \multicolumn{2}{|c|}{ Disentangled } \\
\hline & \multicolumn{4}{|c|}{$E W$} & \multicolumn{4}{|c|}{$E W$} \\
\hline Frequency & ampl \pm s.e. & f.v. & ampl \pm s.e. & f.v. & ampl \pm s.e. & f.v. & ampl \pm s.e. & f.v. \\
\hline$f_{\text {rot }}$ & $0.30 \pm 0.03$ & & $0.29 \pm 0.03$ & & $0.61 \pm 0.02$ & & $0.35 \pm 0.02$ & \\
\hline$f_{1}$ & $0.10 \pm 0.03$ & & $0.15 \pm 0.02$ & & $0.07 \pm 0.02$ & & $0.11 \pm 0.02$ & \\
\hline \multirow[t]{2}{*}{$2 f_{1}$} & $0.11 \pm 0.03$ & $16 \%$ & $0.10 \pm 0.02$ & $23 \%$ & $0.05 \pm 0.03$ & $54 \%$ & $0.04 \pm 0.02$ & $47 \%$ \\
\hline & \multicolumn{4}{|c|}{$\langle v\rangle$} & \multicolumn{4}{|c|}{$\langle v\rangle$} \\
\hline Frequency & ampl \pm s. e. & f. v. & ampl \pm s. e. & f. v. & ampl \pm s. e. & f. v. & ampl \pm s. e. & f. v. \\
\hline$f_{1}$ & $2.13 \pm 0.11$ & & $2.24 \pm 0.10$ & & $2.06 \pm 0.12$ & & $2.21 \pm 0.10$ & \\
\hline$f_{2}$ & $0.55 \pm 0.10$ & & $0.87 \pm 0.09$ & & $0.59 \pm 0.12$ & & $0.95 \pm 0.09$ & \\
\hline$f_{3}$ & $0.32 \pm 0.10$ & & $0.56 \pm 0.09$ & & $0.15 \pm 0.12$ & & $0.41 \pm 0.09$ & \\
\hline $2 f_{1}$ & $0.24 \pm 0.10$ & $44 \%$ & $0.52 \pm 0.09$ & $55 \%$ & $0.35 \pm 0.12$ & $35 \%$ & $0.60 \pm 0.09$ & $55 \%$ \\
\hline
\end{tabular}

In the following we report the results of the frequency analyses on the line diagnostics derived from the profiles obtained with the three different procedures.

\section{Frequency analysis}

We determined the frequencies with an accuracy according to the empirically derived formula $\sigma_{f}=\sigma_{R} / \sqrt{2 N} A T$, with $N$ the amount of datapoints, $T$ the total timespan of the data, $\sigma_{R}$ the standard deviation of the noise and $A$ the amplitude of the variation (see, e.g., Cuypers 1987, for a discussion). For the dominant signal in our data, which span 866 days, this leads to $\sigma_{f}$ of $0.00003 \mathrm{c} \mathrm{d}^{-1}$. For signals with a lower amplitude or when assuming subsets of the data, the accuracy will obviously decrease. This decrease is inversely proportional to the amplitude of the signal (e.g. $\sigma_{f_{2}}=0.0001 \mathrm{~cd}^{-1}$ and $\sigma_{f_{3}}=0.0002 \mathrm{c} \mathrm{d}^{-1}$, see Table 1 for the corresponding amplitudes). We performed CLEAN (Roberts et al. 1987), Lomb-Scargle (Scargle 1982) and PDM (Stellingwerf 1978) analyses.

We considered the variations of the first three velocity moments $\left(\langle v\rangle,\left\langle v^{2}\right\rangle,\left\langle v^{3}\right\rangle\right.$; definition by Aerts et al. 1992), of the equivalent width $(E W)$ and of the normalised flux at each pixel across the profile (Intensity Period Search, IPS) for the two bluest Si III lines. In calculating the moments we took the fixed integration boundaries indicated as vertical lines in Fig. 2. We searched for frequencies in the interval $[0,15] \mathrm{c} \mathrm{d}^{-1}$ with a frequency step of $0.00001 \mathrm{c} \mathrm{d}^{-1}$. As the IPS analysis is very sensitive to the quality of the data, we selected only the subset of 397 spectra with signal-to-noise ratio above 500 for that. As the major part of this subset consists of spectra obtained during the 8 nights of July 1997, we searched for frequencies with a frequency step of $0.001 \mathrm{c} \mathrm{d}^{-1}$.

\subsection{The dominant frequency}

All line diagnostics show one dominant frequency for the three followed procedures: $5.01215 \pm 0.00003 \mathrm{c} \mathrm{d}^{-1}$. The difference between this frequency and $f_{1}=4.99922 \mathrm{c} \mathrm{d}^{-1}$ equals the value of the highest peak in the window function, which is located at $0.01292 \mathrm{c} \mathrm{d}^{-1}$. Therefore, these two frequencies are not distinguishable in our data. The Lomb-Scargle periodogram of the radial velocity (RV) is shown in the left panel of Fig. 3 while phase diagrams of the moments for $f_{1}$ can be found in the left panels of Fig. 4. We note that power also occurs at the first harmonic $2 f_{1}$ in the RV (see Fig. 3).

The $E W$ variations show a dominant frequency at $0.24521 \pm$ $0.00003 \mathrm{c} \mathrm{d}^{-1}$ or its alias $0.23225 \pm 0.00003 \mathrm{c} \mathrm{d}^{-1}$ at a relative amplitude of some $5 \%$ for the disentangled profiles. These two frequency peaks have an amplitude about twice as large as the one for $f_{1}$ in the disentangled profiles, as can be seen in Fig. 3. We find that $f_{1}=4.99922 \mathrm{c} \mathrm{d}^{-1}$ and its first harmonic are only present in the $E W$ variations of the disentangled spectra and not in the profiles derived from the other two procedures. We therefore tentatively conclude that KOREL did a good job in allowing the detection of realistic periodic $E W$ variations with frequency $f_{1}$ at $\sim 3 \%$ level in the line profiles resulting from co-addition of the disentangled profile of the primary to the residual profiles after disentangling. Depending on their effective temperature, $E W$ variations of a few percent are indeed expected and observed in several $\beta$ Cep stars due to their dominant oscillation (De Ridder et al. 2002). It remains to be further studied how well the code would perform in different circumstances, i.e. different oscillations and amplitudes in stars with different rotational velocities.

The phase difference between the RV, $E W$ and light variations is very useful information when it comes to evaluate physical models. We therefore computed these phase differences from a fit with the synodic frequency $f_{1}$ to $E W,\langle v\rangle$ and the KOREL line intensity (LI) described in Paper I. The results are $\phi(\langle v\rangle)-\phi(\mathrm{LI})=0.29 \pm 0.01$ and $\phi(E W)-\phi(\mathrm{LI})=0.10 \pm 0.01$, expressed in $2 \pi$ radians. The former phase difference is in remarkable agreement with the theoretical predictions found for non-adiabatic NRP of low degree and low radial order by De Ridder et al. (2002) for stellar parameters similar to those 

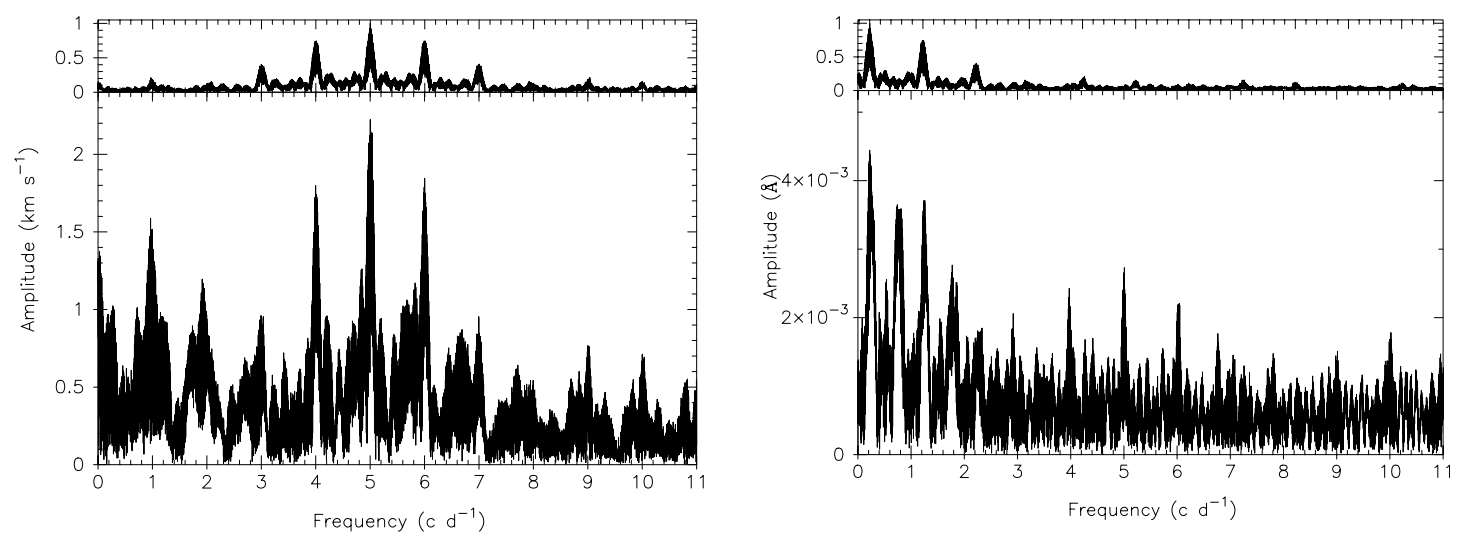

Fig. 3. Lomb-Scargle periodogram of the RV (left) and $E W($ right $)$ derived from the disentangled Si III $4552.654 \AA$ profiles of the primary. On top of each panel, the window function, shifted according to the highest peak in the periodogram, is shown.

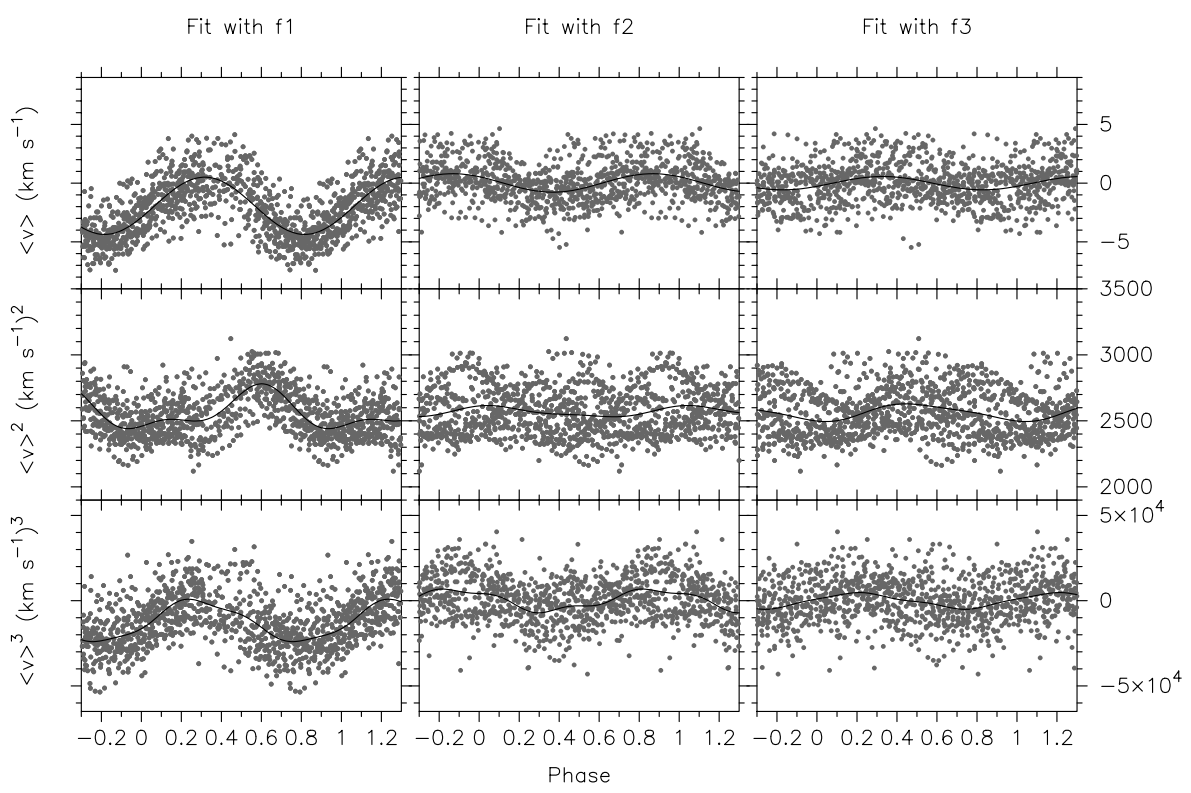

Fig. 4. The first three normalised moments (without prewhitening in case of $f_{1}$; after prewhitening with $f_{1}$ in case of $f_{2}$ and $f_{3}$ ) of the Si III $4552.654 \AA$ line of $\kappa$ Sco, calculated from the disentangled spectra of the primary and folded with the frequencies $f_{1}$ (left), $f_{2}$ (middle) and $f_{3}($ right). The dots are the observations, while the full line is a fit for the indicated frequencies with no, one and two harmonics for respectively $\langle v\rangle,\left\langle v^{2}\right\rangle$ and $\left\langle v^{3}\right\rangle$.

of $\kappa$ Sco. That the $E W$ and LI are in phase and not in antiphase is what can be expected for the Si III triplet subject to NRP for temperatures near $26000 \mathrm{~K}$ (De Ridder et al. 2002).

\subsection{Additional frequencies}

After prewhitening with the dominant frequency $f_{1}$, the highest peaks in the frequency spectrum of the different moments appear in the interval $0-2 \mathrm{~cd}^{-1}$. In particular, a high peak, already clearly visible in the left panel of Fig. 3, occurs in the residual $\langle v\rangle$ data near $0.9 \mathrm{c} \mathrm{d}^{-1}$. This frequency's amplitude in the residuals of $\langle v\rangle$ is about half the one of $f_{1}$. Such a low frequency is not expected for $\mathrm{p}$-modes in $\beta$ Cep stars and its meaning or implication is not clear at this stage of the analysis. We checked that this frequency is not induced by zeropoint differences between the different nights. Such a situation is not uncommon in multiperiodic $\beta$ Cep stars and one tends to continue the analysis with a search for higher frequencies first (see, e.g.,
Aerts et al. 2004a for an extended discussion of such a situation for the $\beta$ Cep star $v$ Eri) before returning to the low-frequency domain, which is also what we do here for $\kappa \mathrm{Sco}$, the more so since we have a complex window function and prewhitening with a frequency near $0.9 \mathrm{c} \mathrm{d}^{-1}$ might introduce changes in the values of the subsequent frequencies.

Candidate frequencies in the expected range of frequencies related to $\mathrm{p}$-modes in $\beta$ Cep stars occur only at low amplitudes slightly above the noise level after prewhitening with $f_{1}$. We stress that any of the frequencies listed below would not be accepted if we were to adopt, e.g., the $4 \times$ signal-to-noise ratio criterion as introduced by Breger et al. (1993) and often used to determine the significance of a frequency nowadays, to our spectroscopic data alone. The reason why we continue the frequency search in quite some detail is twofold:

1. we want to test the differences obtained for the lowamplitude frequencies for the three different procedures 

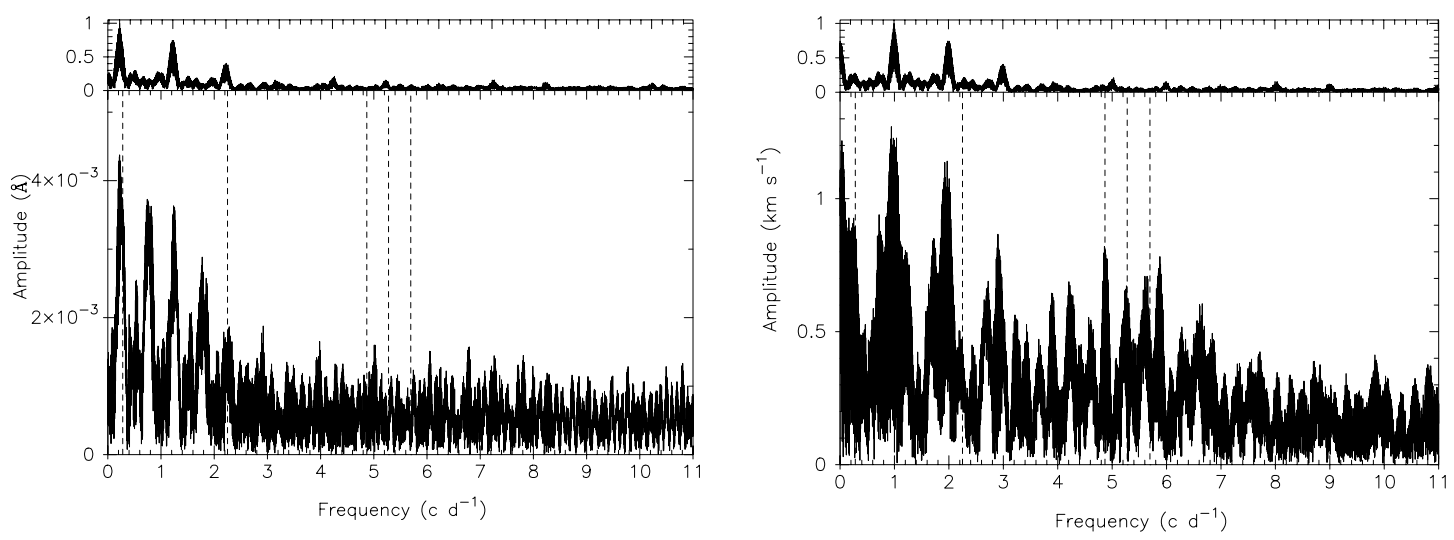

Fig. 5. Lomb-Scargle periodograms of the residuals of the $E W$ (left) and first velocity moment (right) of the disentangled Si III $4552.654 \AA$ profiles of the primary after prewhitening with $f_{1}=4.99922 \mathrm{c} \mathrm{d}^{-1}$. The frequencies reported in the literature are indicated by dashed lines: $4.8678 \mathrm{c} \mathrm{d}^{-1}, 5.2767 \mathrm{c} \mathrm{d}^{-1}$ (Lomb \& Shobbrook 1975), 0.2809 $\mathrm{c} \mathrm{d}^{-1}, 2.2498 \mathrm{c} \mathrm{d}^{-1}$ and $5.6950 \mathrm{c} \mathrm{d}^{-1}$ (WIRE, Cuypers et al. 2004).

employed to derive the profiles of the primary in its rest frame (see Sect. 3);

2. the same candidate frequencies turn out to occur in different, entirely independent datasets of the star, such as our spectroscopy, ground-based photometry (Lomb \& Shobbrook 1975) and space photometry (WIRE, Cuypers et al. 2004), each of which has a complex window function with large aliasing effects.

The Lomb-Scargle periodograms of the $E W$ and $\langle v\rangle$ after prewhitening with $f_{1}$ are given in Fig. 5. Additional ones after subsequent stages of prewhitening as well as additional IPS analyses are provided in Uytterhoeven (2004), $\mathrm{PhD}$ Thesis ${ }^{2}$, to which we refer for a more detailed discussion. We summarise the results of the frequency analyses as follows. Besides the dominant frequency $f_{1}$ and its first harmonic, we find the frequencies reported in the literature $f_{2}=4.8678 \mathrm{c} \mathrm{d}^{-1}$ and $f_{3}=5.695 \mathrm{c} \mathrm{d}^{-1}$ or their aliases. We can only claim the presence of the latter because they were detected before in independent datasets. Phase diagrams for the three moments for $f_{1}, f_{2}, f_{3}$ are shown in Fig. 4 . The corresponding amplitudes for $\langle v\rangle$ are given in Table 1 . Taking different aliases of $f_{2}$ and $f_{3}$ in the fits leads to amplitudes within the uncertainties listed in the table.

We also detected a frequency in the interval [0.227;0.281] $\mathrm{cd}^{-1}$ in all datasets, to which we cannot assign the most probable value due to the poor and complex window functions. A physical explanation might be given in terms of the rotational period of the primary. Indeed, in Paper I we estimated the range of the rotational period to be between 3.56 and 3.68 (corresponding frequencies between $0.272 \mathrm{c} \mathrm{d}^{-1}$ and $0.281 \mathrm{c} \mathrm{d}^{-1}$ ), which is consistent with the derived interval.

The variations in the moments of the disentangled profiles show stronger variability at low frequencies (between 0-2 $\mathrm{c} \mathrm{d}^{-1}$ ) than those of the original and secondary removed profiles. Moreover, $f_{2}$ (in case of the $4552.678 \AA$ profile) and $f_{4}$ do not contribute to the variability derived from the disentangled spectra, while these frequencies do appear in the other

\footnotetext{
${ }^{2}$ Available from http://www.ster.kuleuven.ac.be/pub/ uytterhoeven_phd/
}

two sets of profiles. We must therefore conclude that KOREL disentangling affects the low-amplitude LPV of $\kappa$ Sco. The complex frequency spectrum of $\kappa$ Sco implies that this star is not the best target to derive the consequences of spectral disentangling for the interpretation of LPV in full detail. In order to synthesize the advantages of disentangling as an intermediate step in the interpretation of the LPV, simulations of theoretically generated profiles for multiperiodic oscillations of very different kinds are needed. Such a study is beyond the scope of our current paper, but our experience obtained from $\kappa$ Sco constitutes a good starting point for such a detailed simulation study.

In the following we will work with $f_{1}=4.99922 \mathrm{c} \mathrm{d}^{-1}$ and with $f_{\text {rot }}, f_{2}, f_{3}$. It is important for the reader to realise that we cannot assign definitive values to the latter three frequencies because none of the available datasets allow us to discriminate between the true and alias values. It is therefore evident that any interpretation of the variability with $f_{1}$ on the one hand, and with $f_{\text {rot }}, f_{2}, f_{3}$ on the other hand will be at a very different level of confidence. This has to be kept in mind in evaluating the results reported in the following section.

\section{Interpretation of the line-profile variability}

\subsection{Multiperiodic oscillations}

First we assume the observed LPV to be caused entirely by oscillations with frequencies $f_{1}, f_{2}$ and $f_{3}$. We used a combination of several mode-identification techniques to identify the associated pulsation modes.

By means of the phase and amplitude distributions across the LP (IPS Method, see Telting \& Schrijvers 1997), given in Fig. 6, we assigned wavenumbers $(\ell, m)$ to each of the frequencies $f_{1}, f_{2}, f_{3}$. According to the relationships between $\ell(|m|)$ and the phase difference $\Delta \Psi_{f}\left(\Delta \Psi_{2 f}\right)$ derived by Telting \& Schrijvers (1997) we find that all three modes have degrees below 5 (see Table 2).

We subsequently generated theoretical LP time series, following Telting \& Schrijvers (1997) and Schrijvers \& Telting (1999), for the main frequency $f_{1}$ by considering the different sets of wavenumbers resulting from Table 2 and compare them with the observed ones in Fig. 7. We find good agreement 

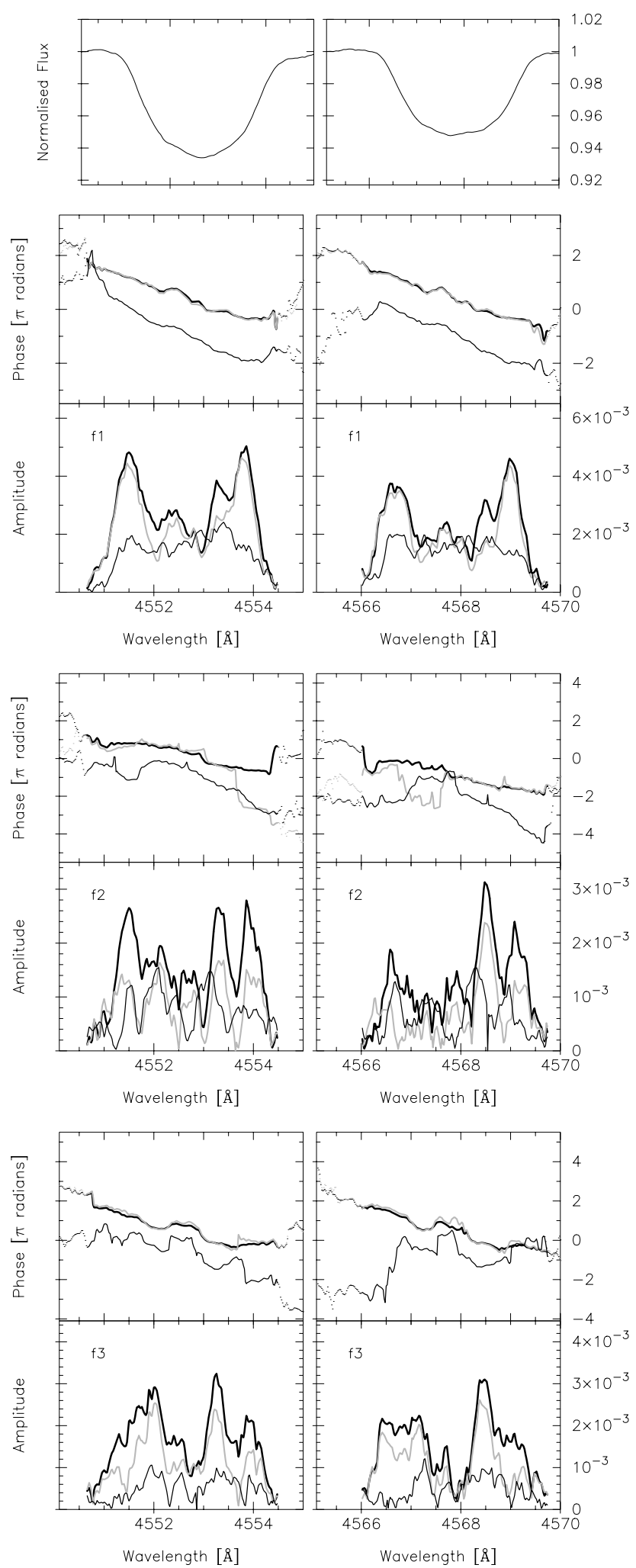

Fig. 6. IPS diagrams for $f_{1}, f_{2}$ and $f_{3}$ and their first harmonic, calculated from the Si III $4552.654 \AA$ (left) and $4567.874 \AA$ (right) line of the disentangled profiles. The top panel shows the average line profile. Each of the three sub-panels shows the distribution of the phase (expressed in $\pi$ radians) and of the amplitude across the line profile. Thick black line: single-sinusoid fit with indicated $f$; light gray line: triple-sinusoid fit with $f_{1}, f_{2}$ and $f_{3}$; thin black line: single-sinusoid fit with $2 f$. For clarity the phase distribution across the line profile for $2 f$ is plotted with an offset.

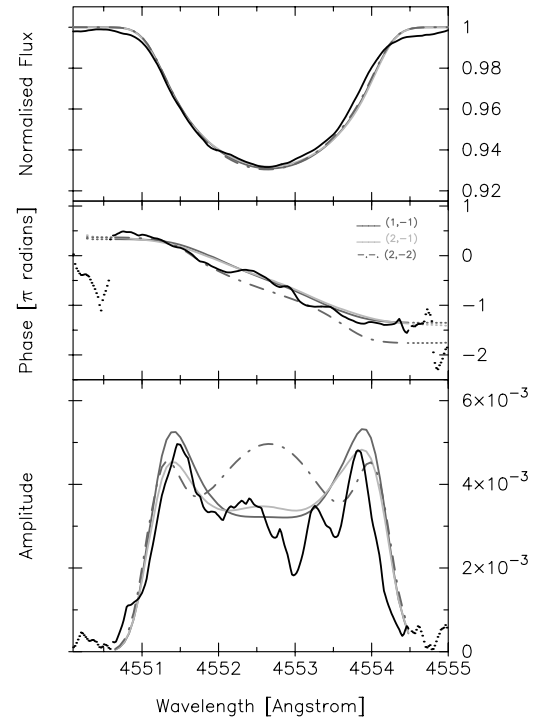

Fig. 7. Mean profile and phase and amplitude diagrams for $f_{1}$ centered at $4552.654 \AA$. The solid black line represents the diagnostics derived from the disentangled spectra. The other lines are derived from theoretically calculated profiles, with different $(\ell, m)$ values: $(1,-1)$ (dark gray solid line); $(2,-1)$ (light gray solid line); $(2,-2)$ (dark gray dashed-dotted line). The phases are given in $\pi$ radians.

Table 2. Blue-to-red phase differences of $f$ and its first harmonic together with estimates for $\ell$ and $m$ for $f_{1}, f_{2}$ and $f_{3}$ (Telting \& Schrijvers 1997). The phase differences $\Delta \Psi$ are given in $\pi$ radians. Due to the bad quality of the IPS diagnostics associated to $2 f_{3}$ (see Fig. 6), we cannot provide information on the $m$-value of this mode.

\begin{tabular}{lllll}
\hline \hline Frequency & $\Delta \Psi_{f}$ & $\Delta \Psi_{2 f}$ & $\ell \in$ & $|m| \epsilon$ \\
\hline$f_{1}$ & 2.5 & 4.5 & {$[2,4]$} & {$[0,2]$} \\
$f_{2}$ & 2.5 & 3 & {$[2,4]$} & {$[0,2]$} \\
$f_{3}$ & 3 & - & {$[2,4]$} & - \\
\hline
\end{tabular}

between the observed and theoretical line diagnostics for the $(1,-1)$ or $(2,-1)$ solution. Despite our massive exploration of the free pulsation parameter set, the theoretical models do not explain the amplitude distribution in all of its details, nor the slightly asymmetric mean profile. This is not suprising, given that $f_{\text {rot }}, f_{2}$ and $f_{3}$ contribute to the LPV albeit at low amplitude while we averaged them out. Despite this shortcoming, we can easily exclude zonal modes and the $\ell=2,|m|=2$ mode, as well as the higher degrees.

For the additional lower-amplitude modes we can only narrow down the intervals of $\ell$ and $|m|$ (see Fig. 8): the $f_{2}$ mode, respectively $f_{3}$ mode, is most likely a tesseral or a sectoral one with $2 \leq \ell \leq 3$, respectively $3 \leq \ell \leq 4$. More precise identifications are not possible because the amplitude and phase behaviour is very similar for different combinations (see also Aerts et al. 2003b for another example of such a situation in the $\beta$ Cep star $16 \mathrm{Lac})$.

In addition, we also used the Moment Method (MM) by Briquet \& Aerts (2003). To identify the mode corresponding to $f_{1}$, we removed the contribution of the low-amplitude variability by averaging out all the observed profiles in phase bins of 0.025 of the oscillation cycle and we worked with 


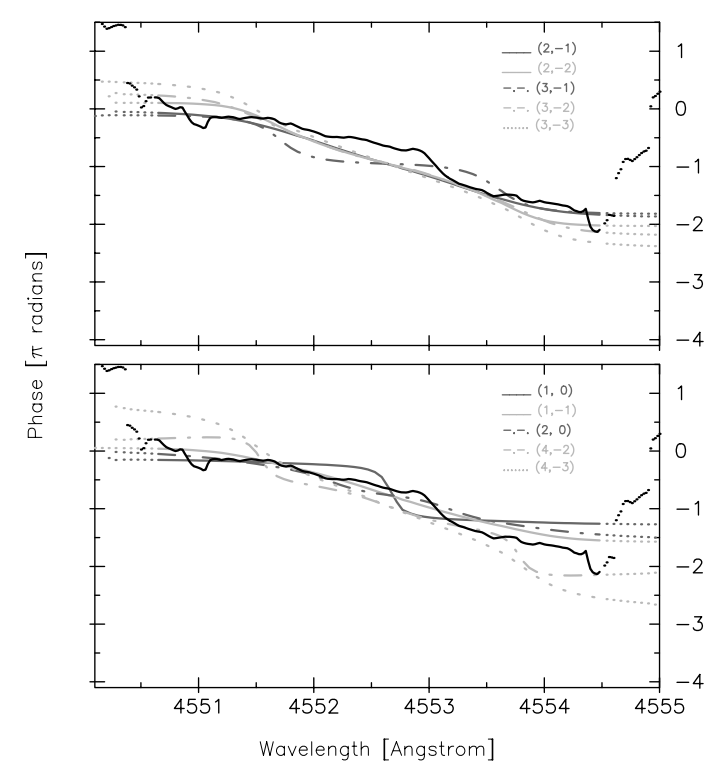

f3

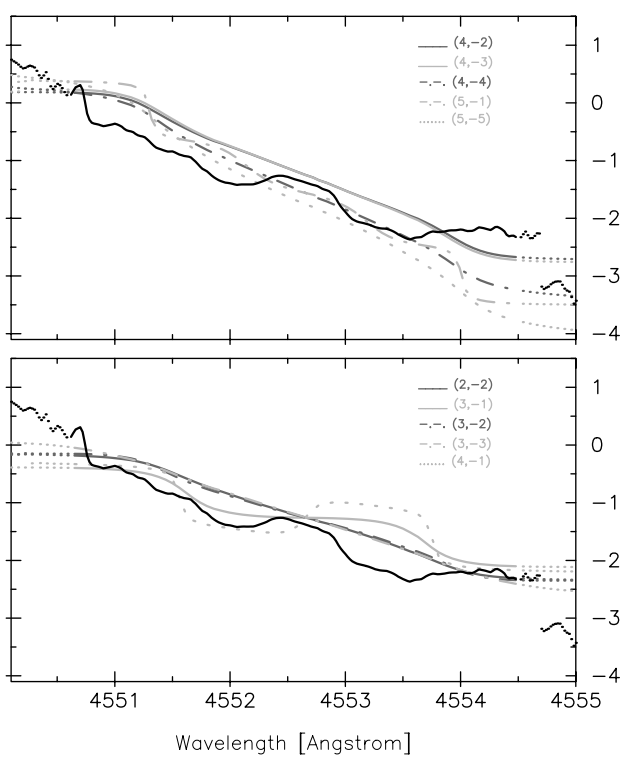

Fig. 8. Phase distribution across the $4552.654 \AA$ profile for sinusoidal fits with $f_{2}$ (left) and $f_{3}$ (right). The solid black line represents the diagnostics derived from the disentangled spectra. The other lines are derived from theoretically calculated profiles, with different $(\ell, m)$ values as indicated. The phases are expressed in terms of $\pi$ radians.

these 40 averaged observed profiles. Their first three velocity moments are shown in Fig. 9. This figure shows clearly the presence of the harmonic $2 f_{1}$, which is confirmed by the Fourier transform on the moments. The amplitudes of the first moment computed from the averaged spectra are respectively $2.20 \mathrm{~km} \mathrm{~s}^{-1}$ and $0.47 \mathrm{~km} \mathrm{~s}^{-1}$ for $f_{1}$ and $2 f_{1}$. The occurrence of harmonics and/or coupling terms is not exceptional for $\beta$ Cep stars, see examples in Heynderickx (1992), Mathias et al. (1994), Aerts et al. (1995), Handler et al. (2004) and Aerts et al. (2004a).

We calculated the discriminant $\Sigma$ (see Briquet \& Aerts 2003 , for the definition and usage) assuming a stellar mass and radius of $M=11 M_{\odot}$ and $R=7 R_{\odot}$ leading to $K=0.095$ for $f_{1}$. For each tested $(\ell, m, i)$, the oscillation amplitude is fixed by the condition that the theoretical amplitude of the first moment has to be equal to the observed one. In the case of $\kappa$ Sco the observed amplitudes exclude $\ell$-values higher than $\ell>3$ for the main mode (see criterion derived in Briquet \& Aerts 2003), which is a lower upper limit than from the IPS analysis.

The MM is implemented for a linear pulsation theory so that it assumes the first moment to be well described by a single cosine function. In spite of the presence of $2 f_{1}$, we performed a mode identification by considering only $f_{1}$. This is justified because the amplitude of the harmonic is about 4 times lower than the one of $f_{1}$. This type of approach turned out to be valid for the $\beta$ Cep star BW Vul, for which the relative contribution of the first harmonic to the radial-velocity variation is also a factor near $1 / 4$ of the one of the frequency itself (Aerts et al. 1995). The results of the MM are given in Table 3. The lower the discriminant $\Sigma$, the better the agreement between theoretical and observed moment values. We conclude that $f_{1}$ corresponds to a prograde mode with $(\ell, m)=(2,-1)$ or $(1,-1)$. This is in perfect agreement with the results found from the IPS Method.
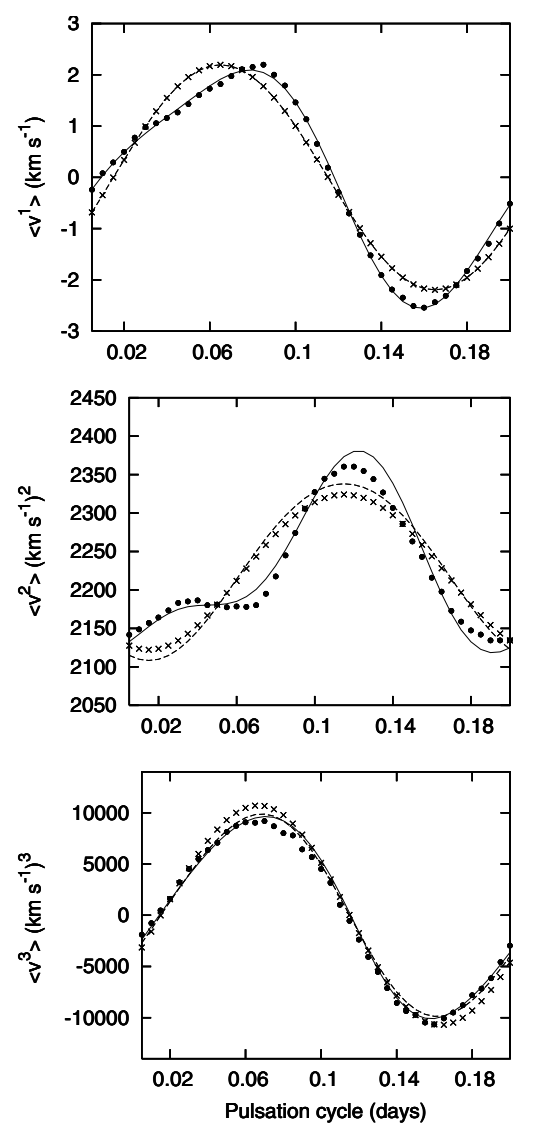

Fig. 9. The first three velocity moments (dots) computed from 40 averaged spectra in the region near Si III $4552.654 \AA$ over the pulsation cycle of $f_{1}=4.99922 \mathrm{c} \mathrm{d}^{-1}$. The crossed and dashed lines correspond respectively to theoretical moments computed for the first two best solutions of the upper part of Table 3 . The full line corresponds to the best solution for a model with $f_{1}$ and $2 f_{1}$ (indicated in bold in Table 4). 
Table 3. List of the five lowest values of the discriminant $\Sigma$ (in $\mathrm{km} \mathrm{s}^{-1}$ ) for the mode identification of $f_{1}$ by the MM. $i$ is the inclination angle, expressed in degrees. The amplitude(s) of the radial part of the pulsation velocity $A_{\mathrm{p}}\left(A_{\mathrm{p}}^{\prime}\right)$, the projected rotational velocity $v \sin i$ and the intrinsic LP width $\sigma$ are all expressed in $\mathrm{km} \mathrm{s}^{-1}$.

\begin{tabular}{cccccc}
\hline \hline$(\ell, m)$ & $\Sigma$ & $A_{p}$ & $i$ & $v \sin i$ & $\sigma$ \\
\hline$(\mathbf{2},-\mathbf{1})$ & $\mathbf{9 . 7 2}$ & $\mathbf{2 4 . 0 4}$ & $\mathbf{7 0}$ & $\mathbf{9 6}$ & $\mathbf{4 . 5}$ \\
$(\mathbf{1},-\mathbf{1})$ & $\mathbf{1 0 . 7 3}$ & $\mathbf{1 4 . 3 6}$ & $\mathbf{5 1}$ & $\mathbf{9 6}$ & $\mathbf{4}$ \\
$(1,0)$ & 12.29 & 8.10 & 13 & 95 & 5.5 \\
$(0,0)$ & 13.37 & 11.33 & 14 & 95 & 5.5 \\
$(2,0)$ & 13.97 & 10.24 & 13 & 94 & 10 \\
\hline
\end{tabular}

Table 4. List of the ten lowest values of the discriminant $\Sigma$ for the mode identification of $f_{1}$ and $2 f_{1}$ by the MM. The symbols are similar as those in Table 3.

\begin{tabular}{cccccccc}
\hline \hline$(\ell, m)$ & $\left(\ell^{\prime}, m^{\prime}\right)$ & $\Sigma$ & $A_{p}$ & $A_{p}^{\prime}$ & $i$ & $v \sin i$ & $\sigma$ \\
\hline$(\mathbf{2},-\mathbf{1})$ & $(\mathbf{2},-\mathbf{2})$ & $\mathbf{8 . 3 2}$ & $\mathbf{2 3 . 1 0}$ & $\mathbf{4 . 4 5}$ & $\mathbf{6 9}$ & $\mathbf{9 5}$ & $\mathbf{8 . 5}$ \\
$(1,-1)$ & $(2,-2)$ & 9.29 & 27.64 & 3.94 & 83 & 96 & 4.5 \\
$(2,-1)$ & $(3,-2)$ & 9.39 & 27.64 & 14.55 & 73 & 95 & 8.0 \\
$(2,-1)$ & $(3,-1)$ & 9.77 & 15.49 & 10.77 & 43 & 94 & 10.0 \\
$(1,-1)$ & $(3,-1)$ & 10.07 & 15.79 & 11.60 & 45 & 95 & 8.0 \\
$(1,-1)$ & $(3,-2)$ & 10.16 & 11.25 & 32.40 & 83 & 94 & 10.0 \\
$(1,-1)$ & $(2,-1)$ & 10.50 & 12.53 & 4.80 & 63 & 96 & 5.0 \\
$(2,-1)$ & $(2,0)$ & 10.51 & 15.93 & 5.51 & 38 & 94 & 9.5 \\
$(2,-1)$ & $(2,-1)$ & 10.82 & 17.85 & 4.48 & 60 & 95 & 8.0 \\
$(1,-1)$ & $(1,-1)$ & 11.03 & 12.22 & 2.76 & 66 & 96 & 5.0 \\
\hline
\end{tabular}

Figure 9 compares the theoretical moment values with the observed ones for the two best solutions given in bold in Table 3 .

Subsequently, we performed a mode identification by also considering $2 f_{1}$ as an additional independent mode. The best combinations are listed in Table 4 . We find that $2 f_{1}$ is also a prograde mode in this model. We note that taking $2 f_{1}$ into account leads to a smaller discriminant value as expected (more free parameters) and allows a much better fit to the observed moments, particularly the second one, as illustrated in Fig. 9. We also point out that the simpler assumption of having modes with the same $(\ell, m)$ values for $f_{1}$ and $2 f_{1}$ does not lead to a very good fit and results in discriminant values that are higher than taking only $f_{1}$ into account (two last rows in Table 4 ).

Considering a $(2,-1)$ or $(1,-1)$ mode for $f_{1}$, we checked the parameter space in $(i, v \sin i)$ which leads to a discriminant of the order of the two lowest values given in Table 3. This allows us to restrict the interval for the equatorial velocity of the star. We found $i \in\left[45^{\circ} ; 90^{\circ}\right]$ and $v \sin i \in[94 ; 100] \mathrm{km} \mathrm{s}^{-1}$. It is well known that the error of the estimate for the inclination from the discriminant is unfortunately large; see De Ridder et al. (2005) for a discussion of the statistical properties and the error propagation of the discriminant.

We also identified the three modes $f_{1}, f_{2}, f_{3}$ simultaneously by using our multiperiodic version of the MM (Briquet $\&$ Aerts 2003) on the 699 moment values of the disentangled spectra. We found that $\left(\ell_{2}, m_{2}\right)$ and $\left(\ell_{3}, m_{3}\right)$ correspond to $(1,-1)$ or $(2,-1)$ with a preference for $(1,-1)$. This result is not compatible with the IPS diagnostics that favour $2 \leq \ell_{2} \leq 3$ and $3 \leq \ell_{3} \leq 4$. We have to conclude that the IPS diagnostics and the MM give different results so that the interpretation of $f_{2}$ and $f_{3}$ in terms of a stellar pulsation model remains insecure.

\subsection{A dominant oscillation in combination with rotational modulation}

The presence of spots, due to either temperature or abundance inhomogeneities, in combination with rotation, can also be the cause of the complex low-amplitude LPV superposed on the effects of the main oscillation mode of $\kappa$ Sco. Arguments in favor of such a model are the dominance of $f_{\text {rot }}$ over $f_{1}$ in the $E W$ (see Table 2), which is not expected for pure NRP, and the shape and behaviour of the colour-diagrams of $\kappa$ Sco (Lomb \& Shobbrook 1975, their Fig. 9), which cannot be simply interpreted as the results of temperature and radius changes of an oscillation, as noted by the latter authors. Rotational modulation occurs for the prototype of the $\beta$ Cep stars (Telting et al. 1997) and also for V2052 Oph, which is a He-strong $\beta$ Cep star (Neiner et al. 2003). These two stars are confirmed magnetic variables, while no magnetic field has been detected for $\kappa$ Scorpii.

An approach to model surface spots is based on the idea of an inhomogeneous distribution of (a) certain element(s) on the stellar surface. The surface abundance distribution of elements can be obtained from Doppler Imaging (DI), which inverts rotationally modulated LPVs into a two-dimensional abundance distribution. Below we present the modelling of the Si distribution on the surface of $\kappa$ Scorpii, whereby the surface temperature and gravity were kept fixed.

The DI technique was applied by using a code called INVERS11, implemented and kindly made available to us by Prof. N. Piskunov. We refer to Piskunov \& Rice (1993) and Rice (1996), who describe DI methods in general, for more information. For the spectral synthesis of local line profiles, model atmospheres, assuming solar abundances, were calculated with Kurucz's ATLAS9 programs (Kurucz 1992, 1993). The Vienna Atomic Line Database (VALD, Piskunov et al. 1995; Ryabchikova et al. 1999; Kupka et al. 1999) provided the atomic line data we needed. Spectra were synthesized with the program SYNTH (Piskunov 1992). We started with solar abundances ( -4.49 dex for silicon) and adjusted them by comparing observed and calculated spectra.

We used the following input parameters for the construction of the surface map: $T_{\text {eff }}=25000 \mathrm{~K}, \log g=3.8, v \sin i=$ $100 \mathrm{~km} \mathrm{~s}^{-1}$ and $i=70^{\circ}$. Any change of these values within the uncertainties do not affect the results of the mapping (see Briquet et al. 2004 for an evaluation of small changes of the input parameters on the final map).

To remove the contribution of $f_{1}, f_{2}, f_{3}$ to the variability of $\kappa$ Sco we averaged out all the observed profiles in phase bins of 0.025 of the rotational cycle and we worked with these 40 averaged observed profiles. The Si map derived from the Si III $4552.654 \AA$ line is shown in Fig. 10. We obtained very similar maps by using the Si III $4567.872 \AA$ line and by using both lines together. We find that an overabundant spot compared to the rest of the stellar surface is present at the pole. 


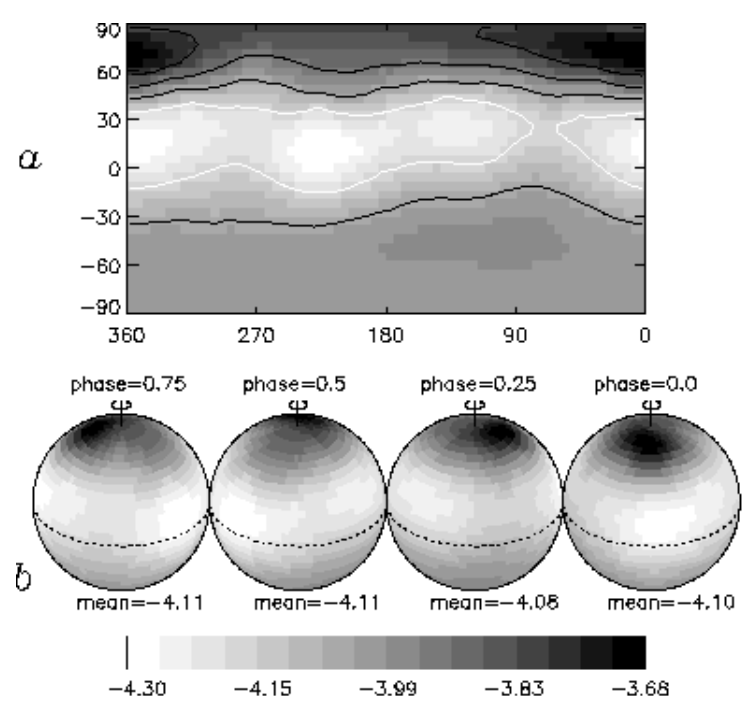

Fig. 10. The Si distribution on the surface of $\kappa$ Sco obtained with INVERS11 from the Si III $4552.654 \AA$ line for $T_{\text {eff }}=25000 \mathrm{~K}$, $\log g=3.8, v \sin i=100 \mathrm{~km} \mathrm{~s}^{-1}$ and $i=70^{\circ}$. a) is the mercator projection of the abundance distribution and comprises contour lines around regions of equal abundance. b) shows the mapped stellar surface in four rotational phases $(0.0,0.25,0.5,0.75)$. Below each phase, values for the mean abundance averaged over the visible part can be found. Dark colour means high, bright colour means low abundance.

The surface part between longitude $-90^{\circ}$ and $-60^{\circ}$ is not visible and consequently we cannot derive the surface abundance near the other pole. We also see the presence of three underabundant spots just above the equator, two of them being more pronounced than the third one. We conclude that an inhomogeneous abundance distribution, after averaging out the dominant oscillation, is compatible with the observed LPVs of $\kappa$ Sco.

Next, we consider LPVs resulting from one dominant prograde NRP mode with frequency $f_{1}$ in combination with a nonhomogeneous intensity distribution at the stellar surface rotating with frequency $f_{\text {rot }}$. In the computations we adopted the value $f_{\text {rot }}=0.2809 \mathrm{c} \mathrm{d}^{-1}$, as suggested in Paper I.

The rotational frequency and radius estimate of $\kappa$ Sco imply that the star rotates at about 20 to $25 \%$ of its break-up velocity. Therefore, the oblateness of the star implies a small gravity gradient, and hence, according to the Von Zeipel theorem, a flux gradient from the equator to the pole. At $25 \%$ of the break-up velocity, the relative flux difference between pole and equator is about $10 \%$ (see, e.g., Fig. 1 in Aerts et al. 2004b), which is of the same order than the relative abundance variation between the equatorial and polar regions found from the Si map. Gravity darkening may therefore explain part of the gradient found in Fig. 10, but not the 3 spots near the equator. Given that we aim only at a modelling of the general characteristics of the observed variability without fine-tuning or optimising the results, and that we do not have a code available that determines the multiperiodic NRP for an oblate star, we assume the surface variations to be caused entirely by a spotted inhomogeneous surface intensity distribution on a spherical star in the calculations described below.

A physical model consisting of a combination of a largeamplitude monoperiodic pulsation and a low-amplitude rotational modulation effect seems quite natural and would not only allow us to give an explanation for the detection of $f_{\text {rot }}, f_{2}$ and $f_{3}$, but also for $f_{4}$ and $f_{5}$ found in respectively groundbased photometry by Lomb \& Shobbrook (1975), and the WIRE satellite data by Cuypers et al. (2004). It is indeed the case that the frequencies $f_{2}, f_{3}, f_{4}$ and $f_{5}$ can be related to $f_{1}$ by means of the rotational frequency, as is already remarked by Lomb \& Shobbrook (1975). We are in the unfortunate situation that we cannot provide exact relations between the frequencies due to our unability to discriminate the frequencies from their aliases.

We generated LPVs for the 699 observational times for $\kappa$ Sco by combined usage of the publicly available code BRUCE (Townsend 1997) which simulates line profiles for a non-radial pulsating star with a code that calculates LPVs for a spherical star with a spotted intensity (kindly made available by Dr. L. Balona). The local intensity within the spots, which, in reality, is determined by the local effective temperature and abundance, is a free input parameter in units of the intensity of the surroundings. The parameters for the oscillation mode were chosen according to our best mode identification for $f_{1}$ and additional estimates thereof obtained in previous sections and Paper I. We considered a simple modulation model with one circular spot in which the intensity has a higher value than the surroundings and with a radius of $30^{\circ}$ at the pole and three circular spots with a lower intensity with the same radius at latitude $20^{\circ}$ equally distant by $120^{\circ}$ in longitude. Such an intensity distribution corresponds to the abundance pattern derived from the DI.

We computed the first moment variation of the synthetic spectra and performed a frequency analysis in the same way as done for the real observations. The whole periodogram of the first moment, before and after prewhitening with $f_{1}$, looks similar to the one of the observed data (left panel Fig. 3 and right panel Fig. 5). In particular, we find a high amplitude at 3 times the used rotational frequency, which occurs near $0.9 \mathrm{c} \mathrm{d}^{-1}$, just as for the real data. This may indicate that this peak is due to 3 spots in a configuration as in the Doppler map shown in Fig. 10. A peak at $f_{2}$ with amplitude of the same order as the observed ones is also present. All this is rather convincing evidence that a rotational modulation effect occurs in $\kappa$ Sco, since this frequency $f_{2}$ was not put in our theoretical model. The frequency $f_{3}$ is hidden in the noise.

Subsequently we calculated the phase distribution across the line profile from the synthetic spectra for a sinusoidal fit with $f_{2}$. The synthetic phase path approaches the bumpy character of the observed phase distribution with similar quality as the one for the multiperiodic oscillation model shown in Fig. 8.

The character of line diagnostics, in particular of moment variations, due to strong abundance spots on a star or due to high-amplitude oscillations in a star are readily distinguishable from each other (see, e.g., Briquet et al. 2004). In the case of $\kappa$ Sco, however, our experiments show that it is not so evident to discriminate between a multiperiodic pulsation model and a model with monoperiodic pulsation and rotational modulation induced by the occurrence of a non-homogeneous surface temperature and/or abundance distribution. The firm proof of rotational modulation would require accurate relations between the detected frequencies and the rotation frequency of the star, 
which we cannot establish due to the limitations of our dataset. In any case, our example of $\kappa$ Sco shows that the presence of frequencies in the range of expected pulsation frequencies for $\beta$ Cep pulsators is not a sufficient condition to conclude the variability to be due to multiperiodic oscillations. In the case of $\kappa \mathrm{Sco}$, the frequencies $f_{1}$ and $f_{\text {rot }}$ are sufficient to explain the occurrence of the frequencies $f_{2}, f_{3}$ in the line diagnostics at low amplitude if we allow for an inhomogeneous surface pattern. On the other hand, it is difficult to exclude firmly the presence of additional pulsation modes with degree $\ell=3$ and with beat frequencies near $f_{\text {rot }}$ for an oblate star with gravity darkening, as this would lead to a similar velocity and temperature distribution on the surface of the star as the proposed models with the temperature or abundance spots. In any case, such a model is also rotationally modulated and so this seems to be a necessary ingredient given that $f_{\text {rot }}$ is dominant in the $E W$ variations.

\subsection{Co-rotating circumstellar structures}

Besides the two mentioned physical models to describe the complex LPV and the observed frequencies of $\kappa$ Sco in terms of oscillations, we consider a third possibility which occurred to us from the following observation. The LPV of $\kappa$ Sco, characterised by the line moments, can also be reconciled with a single frequency $f_{\text {super }}=f_{1} / 17=0.29407 \mathrm{c} \mathrm{d}^{-1}$ and a complicated phase curve. Only a low insignificant peak appears near $f_{\text {super }}$ in the classic periodogram, but a sine fit of $\langle v\rangle$ of $\kappa$ Sco with the frequency $f_{\text {super }}$ fixed and its 25 harmonics leads to a variance reduction of $77 \%$. This is much higher than the result obtained from the fit with $f_{1}, f_{2}, f_{3}, 2 f_{1}$ fixed (see Table 1 ). Note that a good description of the complicated phase curve requires many more free parameters than the multiperiodic solution (53 versus 9) but the phase curves (see Fig. 11) show a good coherence over several years of observations and much lower scatter than the plots for frequencies $f_{2}$ and $f_{3}$ after subsequent prewhitening shown in Fig. 4.

For comparison, a fit with $f_{\text {super }}=0.29407 \mathrm{c} \mathrm{d}^{-1}$ fixed and 3 of its most dominant harmonics (which are $3 f_{\text {super }}, 17 f_{\text {super }}$, and $20 f_{\text {super }}$ ), involving also 9 free parameters, leads to a variance reduction of $65 \%$, which is still higher than the best value of $55 \%$ listed in Table 1 . Similar albeit less convincing results are obtained when fixing the frequencies $f_{1} / 16$ or $f_{1} / 18$ instead of $f_{\text {super }}$. Note also that a fit with $f_{1}, 2 f_{1}, 3 f_{\text {super }}$ and $f_{2}$, again including 9 free parameters, leads to an even better variance reduction of $72 \%$. This latter fit would point to 3 spots in the stellar atmosphere and is basically identical to the model discussed in Sect. 5.2.2. We applied the Bayes Information Criterion (see, e.g., Koen \& Laney 2000) and found that adding more than 9 free parameters to the model would not lead to a significant improvement.

The upper left panel of Figs. 4 and 11 show remarkable similarity with similar figures obtained for the LP variable $\varepsilon$ Per by Harmanec (1999, Figs. 2 and 3). Harmanec (1999) interpreted the variability of the latter star, as well as the variability of the rapid rotator $\zeta \mathrm{Oph}$, in terms of circumstellar structures co-rotating slightly above the photosphere rather than

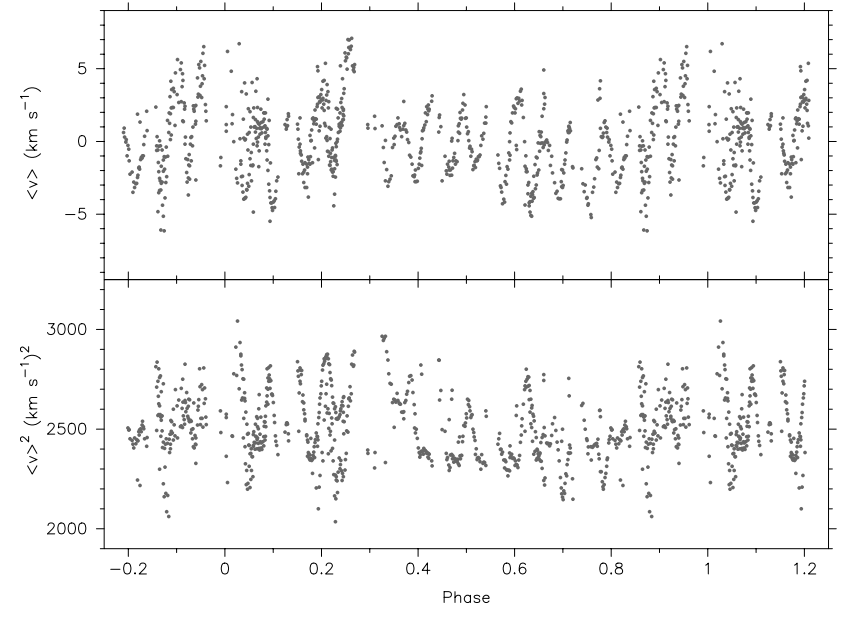

Fig. 11. A phase plot of the first two moments of the disentangled Si III 4552.654 line profiles folded with $f_{\text {super }}=0.29407 \mathrm{c} \mathrm{d}^{-1}$.

multiperiodic oscillations. There is a long and stubborn debate about the correct interpretation of the LPV of rapidly-rotating early-type stars (see, e.g., Baade \& Balona 1994; Harmanec 1989, 1999, and references therein). This debate could also be held for a confirmed $\beta$ Cep star, casting some doubt on the interpretation of $\kappa$ Sco as a NRP star. Indeed, the frequency $f_{\text {super }}$ can be reconciled with the rotational frequency of the star and the LPV can then be qualitatively explained in terms of 17 corotating structures, such as envisioned by Harmanec (1989). Figure 11 shows that the spacing of the structures is quite regular (corresponding to the dominant frequency $f_{1}$ ) but the amplitude and mean value of individual cycles is different (as the need for many harmonics confirms) remaining at the same time stable at various phases of the rotational period over at least a decade. This implies a rather regular spacing but a slightly different strength of the individual putative corotating spokes.

However, we refrain from any more detailed discussion at the moment since it cannot be held on equally quantitative grounds as for the physical models discussed above. The problem is that there exists, unfortunately, no quantitative theoretical description of the model of co-rotating circumstellar structures (see also Harmanec 1999, for details). The first step towards a quantitative description is presented by Clarke (2003), but this author carried out only relatively simple simulations and did not provide a prediction of the amplitudes and phases of the LPV for the circumstellar spokes in different filters and/or spectroscopic data. This is also the reason why we are unable to indicate what kind of additional data would help to test the hypothesis of co-rotating structures further.

For the moment we conclude that the evidence presented in this subsection is not strong enough to reject the oscillatory nature of $\kappa$ Sco, especially since the model which combines rotational modulation superimposed on the $\beta$ Cep oscillation seems to explain the observed variability in a physically adequate way, similarly as for other well studied $\beta$ Cep stars. We point out, however, that a unique identification of all the frequencies of $\kappa$ Sco requires a data set with a longer time base and a better defined window function than the one at hand. 


\section{Summary and discussion}

We have considered $\kappa$ Sco as a test case for the application of KOREL disentangling as an intermediate step in the interpretation of short-period LPV in early-type stars. We investigated its performance by means of a comparison between three sets of profiles obtained from different procedures after orbital subtraction. We find that the LPV due to the high amplitude mode of $\kappa$ Sco is well preserved in all the line diagnostics derived from the KOREL disentangled spectra. This is by no means evident as KOREL was certainly not designed for this purpose and in fact, we violate one of its starting principles: that there be no shape variations in the profiles. Our experiment has shown that the short-term large-amplitude variability is well interpreted as a sort of random noise by the code. It is therefore evident that, in the case of large-amplitude short-term LP variables in close binaries, KOREL disentangling is an extremely useful tool. It would be interesting to carry out a similar performance test of KOREL disentangling on a case study where also the secondary shows obvious LPVs.

As for the low-amplitude variability, we were unable to evaluate the capabilities of KOREL in detail. This is probably due to our inability to derive accurately the low-amplitude frequencies of $\kappa$ Sco. Indeed, we found evidence for three frequencies $f_{\text {rot }}, f_{2}, f_{3}$ besides the dominant frequency $4.99922 \mathrm{c} \mathrm{d}^{-1}$, but these are not significant in the spectroscopic data. We accepted them as real because they were derived from two additional independent photometric datasets (Lomb \& Shobbrook 1975; Cuypers et al. 2004). The derivation and interpretation of these low-amplitude frequencies is somewhat different for the disentangled versus non-disentangled spectra but it is not clear whether the results for the disentangled profiles are more appropriate than for the original ones. For the moment, we therefore have to refrain from claiming KOREL to handle variability with amplitude below $1 \mathrm{~km} \mathrm{~s}^{-1}$ in an appropriate way, which is by no means a negative outcome of our study. A good future testcase to check the validity of KOREL for a multiperiodic non-radial oscillator is $\beta$ Cru (Aerts et al. 1998) although most progress will be achieved by combining the analyses of real datasets with simulations.

The analysis of the LPV of the pulsating primary $\kappa$ Sco, based on large dataset consisting of 699 spectra, covering a time span of 3 years, resulted in a new understanding of the intrinsic variability of the star. We identified the dominant frequency as a prograde NRP mode with spherical wavenumbers $(\ell, m)=(2,-1)$ or $(1,-1)$, whereby the contribution of at least its first harmonic $2 f_{1}$ is also needed to explain the observed variations of the velocity moments. Moreover, we found strong evidence for the presence of a rotational modulation effect in the Si III line profiles. We presented a simple temperature or abundance spot model which allowed us to explain the frequencies $f_{2}$ and $f_{3}$ suggested here and also in the literature before (Lomb \& Shobbrook 1975; Cuypers et al. 2004).

Our detailed analysis of $\kappa$ Sco stresses that the derivation of frequencies in the range of the pulsation frequencies expected for $\beta$ Cep pulsators resulting from Fourier decomposition does not necessarily prove the existence of multiperiodic oscillations. A comparison between observations and a theoretical physical model is needed to check the real cause of the observed frequencies considering all the LP diagnostics. In the present paper we performed such a comparison for a multiperiodic oscillation model and for a modulated monoperiodic oscillation model, keeping in mind that a circumstellar patch model is not straightforward to prove nor disprove. We conclude that $\kappa$ Sco has one dominant NRP mode rotationally modulated by an inhomogeneous surface temperature and/or abundance distribution, as $\beta$ Cep (Telting et al. 1997), a slow rotator, and V2052 Oph (Neiner et al. 2003), a moderate rotator. In all three stars, one dominant frequency occurs in the radial velocity variations while the rotational frequency is dominant in the $E W$ variations. The latter hence played a crucial role in the interpretation of the LPV for these three $\beta$ Cep stars.

As the combination of one NRP mode and inhomogeneities at the stellar surface of a rotating star seems to explain the very complex LP character of $\kappa$ Sco quite well, the question rises if the same model might explain similar variations observed in the complex spectral lines of other moderate rotators among the $\beta$ Cep stars, e.g. $\varepsilon$ Per (De Cat et al. 2000, and references therein), $\alpha$ Vir (e.g. Smith 1985a,b), $\omega^{1}$ Sco (Telting \& Schrijvers 1998; Berdyugina et al. 2003), $v$ Cen (Schrijvers \& Telting 2002). In many of these cases, the frequency spectrum is dominated by one frequency while additional frequencies appear near the noise level as in $\kappa$ Sco. A similar modulated oscillation model as presented in this paper may be interesting to check as an explanation for their complex variability. To do so, however, one needs high signal-to-noise high-spatial and temporal resolution spectroscopy with a time base of years.

Acknowledgements. The authors acknowledge the referee, Dr. G. Handler, for his valuable comments, Dr. P. Hadrava for sharing his code KOREL and for his advice on its use, Prof. N. Piskunov for sharing the codes INVERS11 and SYNTH, Dr. R. Townsend for the code BRUCE and Dr. L. Balona for making some of his codes available. We also thank the Vienna group for putting the Vienna Atomic Line Database at our disposal. This study has benefited greatly from the senior fellowship awarded to P.H. by the Research Council of the University of Leuven which allowed his three-month stay at this university. His research was also supported from the research plans J13/98: 113200004 of Ministry of Education, Youth and Sports and AV 0Z1 003909 and project K2043105 of the Academy of Sciences of the Czech Republic. K.U. is supported by the Fund for Scientific Research - Flanders (FWO) under project G.0178.02 and C.A. and K.L. by the Research Fund K.U. Leuven under grant GOA/2003/04.

\section{References}

Aerts, C., De Pauw, M., \& Waelkens, C. 1992, A\&AS, 266, 294

Aerts, C., Mathias, P., Van Hoolst, T., et al. 1995, A\&A, 301, 781

Aerts, C., De Cat, P., Cuypers, J., et al. 1998, A\&A, 329, 137

Aerts, C., Thoul, A., Daszynska-Daszkiewicz, J., et al. 2003a, Science, 300, 1926

Aerts, C., Lehmann, H., Briquet, M., et al. 2003b, A\&A, 399, 639

Aerts, C., \& De Cat, P. 2003, Space Sci. Rev., 105, 453

Aerts, C., De Cat, P., Handler, G., et al. 2004a, MNRAS, 347, 463

Aerts, C., Lamers, H. J. G. L. M., \& Molenberghs, G. 2004b, A\&A, 418,638

Baade, D., \& Balona, L. A. 1994, in Pulsation, Rotation and Mass Loss in Early-Type Stars, ed. L. A. Balona, H. F. Henrichs, J. M. Le Contel (Dordrecht: Kluwer), IAU Symp., 162, 311 
Berdyugina, S. V., Telting, J. H., Korhonen, H., \& Schrijvers, C. 2003, A\&AS, 406, 281

Breger, M. 1993, in Stellar Photometry - Current Techniques and Future Developments, ed. C. S. Butler, \& I. Elliott (Cambridge Univ. Press), IAU Coll., 136, 106

Briquet, M., Aerts, C., Lüftinger, T., et al. 2004, A\&A, 413, 273

Briquet, M., \& Aerts, C. 2003, A\&AS, 398, 687

Clarke, D. 2003, A\&A, 407, 1029

Cuypers, J. 1987, The Period Analysis of Variable Stars, Academiae Analecta, Royal Academy of Sciences, Vol. 49, No. 3, Belgium

Cuypers, J., Buzasi, D., \& Uytterhoeven, K. 2004, in Variable Stars in the Local Group, IAU Coll., 193, ed. D. W. Kurtz, \& K. Pollard, ASP Conf. Ser., 310, 251

De Cat, P., Telting, J. H., Aerts, C., \& Mathias, P. 2000, A\&AS, 359, 539

De Ridder, J., Dupret, M.-A., Neuforge, C., \& Aerts, C. 2002, A\&A, 385,572

De Ridder, J., Molenberghs, G., \& Aerts, C. 2005, Applied Statistics, 54 , part 2,1

Hadrava, P. 1995, A\&AS, 114, 393

Hadrava, P. 1997, A\&AS, 122, 581

Handler, G., Shobbrook, R. R., Jerzykiewicz, M., et al. 2004, MNRAS, 347, 454

Harmanec, P. 1989, Bull. Astron. Inst. Czechosl., 40, 201

Harmanec, P. 1999, A\&A, 341, 867

Harmanec, P., Uytterhoeven, K., \& Aerts, C. 2004, A\&A, 422, 1013 (Paper I)

Heynderickx, D. 1992, A\&AS, 96, 207

Koen, C., \& Laney, D. 2000, MNRAS, 311, 636

Kupka, F., Piskunov, N. E., Ryabchikova, T. A., et al. 1999, A\&AS, 138, 119

Kurucz, R. L. 1992, Stellar Population of Galaxies, ed. B. Barbuy, \& A. Renzini, IAU Symp., 149, 225
Kurucz, R. L. 1993, ATLAS9, Stellar Atmosphere Programs, CD-ROM No. 13, Smithonian Astrophysical Observatory

Lomb, N. R., \& Shobbrook, R. 1975, MNRAS, 173, 709

Mathias, P., Aerts, C., Gillet, D., \& Waelkens, C. 1994, A\&A, 289, 875

Neiner, C., Henrichs, H. F., Floquet, M., et al. 2003, A\&AS, 411, 565

Pamyatnykh, A. A. 1999, Acta Astr., 49, 119

Pamyatnykh, A. A., Handler, G., \& Dziembowski, W. A., et al. 2004, MNRAS, submitted

Piskunov, N. E., Kupka, F., Ryabchikova, T. A., et al. 1995, A\&AS, 112,525

Piskunov, N. E., \& Rice, J. B. 1993, PASP, 105, 1415

Piskunov, N. E. 1992, in Stellar magnetism, ed. Yu. V. Glagolevskij, \& I. I. Romanyuk (St Petersburg: Nauka), 92

Rice, J. B. 1996, IAUS, 176, 19

Roberts, D. H., Lehar, J., \& Dreher, J. W. 1987, AJ, 93, 968

Ryabchikova, T. A., Piskunov, N. E., Stempels, H. C., et al. 1999, Phys. Scripta, Vol. T83, 162

Santolaya-Rey, A. E., Puls, J., \& Herrero, A. 1997, A\&A, 323, 488

Scargle, J. D. 1982, ApJ, 263, 835

Schrijvers, C., \& Telting, J. H. 1999, A\&AS, 342, 453

Schrijvers, C., \& Telting, J. H. 2002, A\&AS, 394, 603

Shobbrook, R., \& Lomb, N. R. 1975, MNRAS, 156, 181

Smith, M. A. 1985a, ApJ, 297, 206

Smith, M. A. 1985b, ApJ, 297, 224

Stellingwerf, R. F. 1978, ApJ, 224, 953

Telting, J. H., Aerts, C., \& Mathias, P. 1997, A\&AS, 322, 493

Telting, J. H., \& Schrijvers, C. 1997, A\&AS, 317, 723

Telting, J. H., \& Schrijvers, C. 1998, A\&AS, 339, 150

Townsend, R. H. D. 1997, MNRAS, 284, 839

Uytterhoeven, K., Aerts, C., De Cat, P., et al. 2001, A\&A, 371, 1035

Uytterhoeven, K. 2004, Ph.D. Thesis, KU Leuven, Belgium 